\title{
A Novel Fuzzy Logic-Based Medical Expert System for Diagnosis of Chronic Kidney Disease
}

\author{
Jimmy Singla $\mathbb{D}^{1},{ }^{1}$ Balwinder Kaur, ${ }^{1}$ Deepak Prashar, ${ }^{1}$ Sudan Jha ${ }^{(D)}{ }^{1}$ \\ Gyanendra Prasad Joshi $\mathbb{D}^{2},{ }^{2}$ Kyungyun Park $\left(\mathbb{D},{ }^{3}\right.$ Usman Tariq, ${ }^{4}$ and Changho Seo ${ }^{3}{ }^{3}$
}

\footnotetext{
${ }^{1}$ School of Computer Science and Engineering, Lovely Professional University, Phagwara, Punjab, India

${ }^{2}$ Department of Computer Science and Engineering, Sejong University, Seoul 05006, Republic of Korea

${ }^{3}$ Department of Convergence Science, Kongju National University, Gongju 32588, Republic of Korea

${ }^{4}$ College of Computer Science and Engineering, Prince Sattam bin Abdulaziz University, Saudi Arabia
}

Correspondence should be addressed to Gyanendra Prasad Joshi; joshi@sejong.ac.kr and Changho Seo; chseo@kongju.ac.kr

Received 7 March 2020; Accepted 24 April 2020; Published 5 June 2020

Academic Editor: Ali Kashif Bashir

Copyright (C) 2020 Jimmy Singla et al. This is an open access article distributed under the Creative Commons Attribution License, which permits unrestricted use, distribution, and reproduction in any medium, provided the original work is properly cited.

\begin{abstract}
Chronic kidney disease is a life-threatening complication. Primary diagnosis and active control avoid its progression. To increase the life span of a patient, it is necessary to detect such diseases in early stages. In this research paper, design and development of a fuzzy expert system (FES) to identify the current stage of chronic kidney disease is proposed. The proposed fuzzy rule-based expert system is developed with the help of clinical practice guidelines, database, and the knowledge of a team of specialists. It makes use of input variables like nephron functionality, blood sugar, diastolic blood pressure, systolic blood pressure, age, body mass index (BMI), and smoke. The normality tests are applied on different input parameters. The input variables, i.e., nephron functionality, blood sugar, and BMI have more impact on the chronic kidney disease as shown by the response of surface analysis. The output of the system shows the current stage of patient's kidney disease. Totally 80 tests were performed on the FES developed in this research work, and the generated output was compared with expected output. It is observed that this system succeeds in $93.75 \%$ of the tests. This system supports the doctors in assessment of chronic kidney disease among patients. The detection of chronic kidney disease is a serious clinical problem that comprises imprecision, and the use of fuzzy inference system is suggested to overcome this issue. The proposed FES is implemented in the MATLAB.
\end{abstract}

\section{Introduction}

Chronic kidney disease or chronic renal disorders arise due to the lack of functionality of nephrons in the kidney. It basically occurs when the kidney stops its functionality like the maintenance of $\mathrm{pH}$ value, water, and salt in the blood. The kidney is the natural filter of blood and it discharges the wastage from the body in the form of urine. This disease can also lead to the damage of other neighbor organs of a kidney.

Nowadays, due to increase in population and unhealthy lifestyle, number of patients with renal disorder is increasing $[1-3]$. It is tough for individual to recover from every kidney disease [5]. The detection of kidney disease and disorder can be done by studying the features extracted from an acquired image. The image-processing techniques are applied on magnetic resonance images of total kidney volume [6]. The registration is performed on the selected portion of an image to increase the accuracy [7].The optimal path is detected during the segmentation of magnetic resonance images of kidneys for diagnosis of Chronic Kidney Disease (CKD) [8]. Computer aided diagnostic systems are used to examine the kidney features for the early identification of kidney diseases $[9,10]$. Certain test, like F-test, is applied to find out the dependency of features on the kidney area [11]. Ultrasonography images are also used for detection of stages of CKDs. The stages of CKD are predicted by using machine learning techniques [12-15]. The comparisons have been made between the performances of different systems developed by using various algorithms of machine learning [16]. Intelligent models are designed by using different 
methods of artificial neural networks, such as generalized feed forward neural network, back-propagation neural network, and modular neural network [17]. The Naive-Bayes method is also used to classify the various stages of CKD [18]. An approach is designed for detection of disease at initial stage by training the neural network with some amount of training data [19-21]. Further, the data mining algorithms and techniques are used for CKD detection $[22,23]$. Ensemble learning is used to clean the data that are acquired and to fill the values which are missing in that particular dataset to recalculate the different stages of CKD, and hence, the performance of the system is increased with this step [24]. The decision support systems are also built by using the information or dataset of CKD [25].

Early detection of the disease permits the doctor to suggest the required measure so that the risk may reduce [26]. The ranks are given to all the risk factors that extremely affect the kidney [27, 28]. Renal illness is a public health problem across the nation. In Taiwan, there are over 1,100,000 patients living with $\mathrm{CKD}$ and this number is increasing gradually. Moreover, the data from United States Renal Data System (USRDS) stated that Taiwan has extreme occurrence of end stage kidney disease across the world $[29,30]$.

In India, the death rate is increasing rapidly due to various chronic diseases, and $\mathrm{CKD}$ is one of the major diseases among them. The average age of a person that suffers from CKD is from 18 to 98 years in which the number of male patients is more than females [31]. The CKD is not detected easily till it grows due to its asymptomatic nature, and it results in decrease in the opportunities for prevention of the disease [32,33]. It is very important to detect and treat CKD in early stages to avoid kidney failure [34]. It is reported that this problem can be mitigated by developing fuzzy logic-based expert system [35].

The main objective of this work is to develop a medical expert system for diagnosis of chronic kidney disease by using fuzzy logic. The aim is to assist doctors in diagnosis of patients suffering from chronic kidney disease. Fuzzy logic is a rule-based logic that deals with imprecise concepts. It is a many-valued logic that can combine human heuristics into computer-assisted decision-making. The expert system is a computer program which is derived from the artificial intelligence and can be used in real-time applications [36]. Over $70 \%$, this domain is used to solve the real-life applications [37]. The expert systems are used for diagnosis of various diseases. The main idea behind expert system is that the knowledge from the experts of a specific disease will be transferred to the computer system [38]. The knowledge stored in the computer system can be used by the user for specific task. After that, the system will conclude the result by doing various estimations.

The fuzzy logic-based system is having three blocks and the fuzzy logic-based system used here is Mamdani type fuzzy model, all three blocks are discussed as follows:

(1) Fuzzification: It is the process of transforming the crisp values into the fuzzy inputs. The crisp values are inputs measured by the sensors or the values given by the users [39].
(2) Inference mechanism: In the inference mechanism, the system maps rules satisfy input given by user and it gives an output.

(3) Defuzzification: This is the last step in the system. In defuzzification process, a single number from the output of the aggregated fuzzy set is obtained.

Response surface methodology is a set of precise and numerical procedures that are supportive for exhibiting and analysimg issues wherever an interest response obtains the impact of various parameters, and also the aim is to maximise such response [39-41].

The research works discussed above provide fruitful information about the applications of expert systems for medical diagnosis and especially for the diagnosis of chronic kidney disease. The primary risk factors or causes of the chronic kidney disease have been used as the input variables in the development of a proposed medical expert system. After the processing of all blocks of fuzzy logic, it generates an output. In this developed medical expert system, the output is obtained according to the health of a patient who is suffering from this disease.

The main limitations of this system are that the number of rules and input variables can be increased to get the more accurate result and also this system does not have capability to adapt itself according to the environment or to train itself from new examples.

The rest of the paper is organized as follows.

In Section 2, the related work is explained briefly. In Section 3, some established theories about fuzzy logic and notations used in the proposed system are discussed. In Section 4, the methodology for the proposed work including architecture of input-output parameters is described. The normality tests performed on different input variables are also shown in this section. The performance of the proposed system in terms of sensitivity, specificity, precision, and accuracy is evaluated in Section 5. In Section 6, the fuzzy expert system developed in this work along with ideas about future work is discussed. Finally, the work is concluded in Section 7.

\section{Related Work}

Kubota et al. [5] proposed a methodology by using imageprocessing technique in which the detection of the kidney failure of a patient has been done in the early stage based on information of kidney region. The required information is gathered from the CT image of kidney. The image of kidney is segmented into number of regions to collect correct and accurate information from it. Tang et al. [7] projected a method of registration known as intrasubject registration by using the local MI maximization. In this method, the registration is not done on the unified volumes that were acquired from the abdomen region of the patient's body. It will be accomplished only on the selected areas of kidney, which are affected by the disease. This method also increases the accuracy of performing registration. Bommanna Raja and Madheswaran [8] developed a computer aided system that examined the various features which are unconstrained and 
also independent of kidney's area. These examined features are used in the developed system to examine the disease. Rovenţa and Roşu [37] developed a medical expert system to diagnose twenty-seven different kidney diseases from nine different categories. The identification of kidney disease is done by taking the symptoms that can be seen in clinical examination and the result evaluated in the laboratory test. The system was developed by using Visual Prolog 5.2 and helps the doctor to diagnose the disease more accurately.

Shen et al. [29] developed a low cost method for the patients of dialysis. This method is used to identify the risks of cardiovascular disease (CVD) on the chronic kidney disease (CKD). ECG features and heart rate variability of a patient are considered in this system. It uses the decisionbased neural network structure for the feature fusion. The overall accuracy of developed method for cardiovascular disease on chronic kidney disease is $71.07 \%$. Adam and Hashim [19] used the artificial neural network technique for the detection of kidney problems in the initial stage. This method predicts various symptoms of kidney problem and then compares these symptoms with the mental behaviour of an individual. The artificial neural network is first trained by giving training samples, and then the testing procedure is done for the same. Chw et al. [17] designed intelligence models by using the various technologies of artificial neural network such as back-propagation network (BPN), generalized feed forward neural networks (GRNN), and modular neural network (MNN). These methodologies are developed to detect the chronic kidney disease at initial stages. These three models are compared with each other based on accuracy and effectiveness and also deployed on the Google Cloud platform. The constructed models help to avoid the chronic renal disorders in the beginning of the disease or in the introductory stages. Ahmed et al. [42] proposed a fuzzy expert system for the diagnosis of chronic kidney disease. The seven inputs were considered, and by using the values of these inputs, the membership functions are generated. The rules are further generated, and Matlab software is used to implement this research work. The output is predicted by expert system and it gave similar results as the human expert can give.

\section{Notations and Preliminaries}

The notations and preliminaries used in this work are as follows [43].

3.1. Crisp Sets. The crisp set or the classical set is defined as the assemblage or group of elements $x \in X$ that can be finite. For example, to define the set if elements are larger than 5 , the membership function can be defined as follows:

$$
X=\{x \mid x>5\},
$$

where $X$ is the set of positive integers.

3.2. Fuzzy Sets. If $X$ is the set of elements denoted by $x$, then a fuzzy set $A$ in $X$ is the set of ordered pair and is represented by

$$
A=\frac{\left(x, \mu_{A}(x)\right)}{x \in X},
$$

where $\mu_{A}(x)=$ membership function of $x$ in $A$.

3.3. Fuzzy Numbers. A fuzzy number $U$ is convex. A normalized fuzzy set $U$ of the real line $R$, such that

$$
\mu_{U}(x): R \longrightarrow[0,1], \forall x \in R,
$$

where $\mu_{U}=$ membership function of fuzzy set.

There are many fuzzy numbers that exist. But in this work, only trapezoidal fuzzy numbers are used.

3.4. Trapezoidal Fuzzy Number (TrFN). Assume an arbitrary trapezoidal fuzzy number $\widetilde{U}=(p, q, r, s)$. The membership function of $\widetilde{U}$ is represented by $\mu_{\tilde{U}}$ and is given by

$$
\mu_{\tilde{U}}(x)= \begin{cases}0, & x \leq p, \\ \frac{x-p}{q-p}, & p \leq x \leq q, \\ 1, & q \leq x \leq r, \\ \frac{s-x}{s-r}, & r \leq x \leq s, \\ 0, & x \geq s .\end{cases}
$$

\section{The Proposed Methodology}

The methodology applied in this research work is shown in Figure 1.

The expert system has one layer. As shown in Figure 2, this layer detects the stage of CKD based on various input variables. The proposed expert system is explained mathematically as follows:

$$
\mu_{\mathrm{RC}, \text { layer1 }}=\operatorname{MFIS}\left[\mu_{\mathrm{NF}} \mu_{\mathrm{BS}}, \mu_{\mathrm{DBP}}, \mu_{\mathrm{SBP}}, \mu_{\mathrm{Age}}, \mu_{\mathrm{BMI}}, \mu_{\mathrm{Smoke}}\right] .
$$

4.1. Selection of Input and Output Parameters. The attributes should be selected carefully so that classification of patients can be done perfectly $[44,45]$. The details of seven input variables used in this research work are described as follows.

4.1.1. Nephron Functionality (NF). The nephron functionality is the proportion of active nephron in the kidney [42]. The nephron functionality is in safe zone if the value is greater than 0.47 . However, the functionality is moderately risky if the value is in between 0.3 to 0.5 and it is very-risky if it is below 0.35 .

4.1.2. Blood Sugar (BS). The amount of glucose available in the blood refers to the blood sugar. It is composed of three 


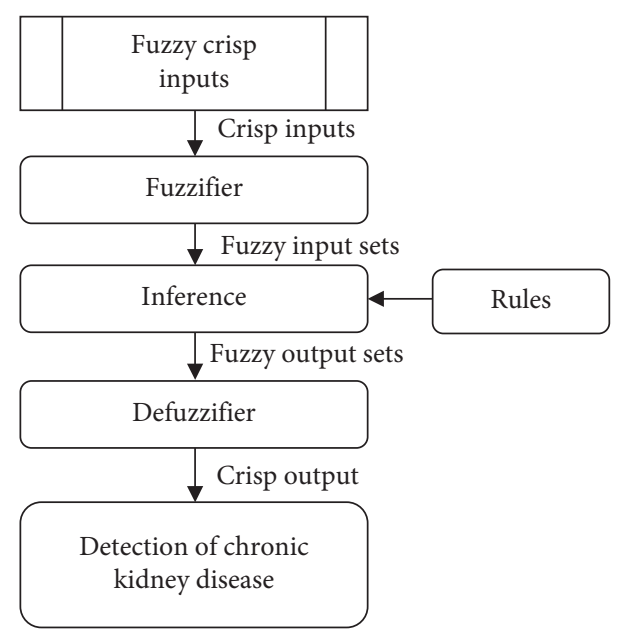

FIGURE 1: Methodology of the proposed expert system.

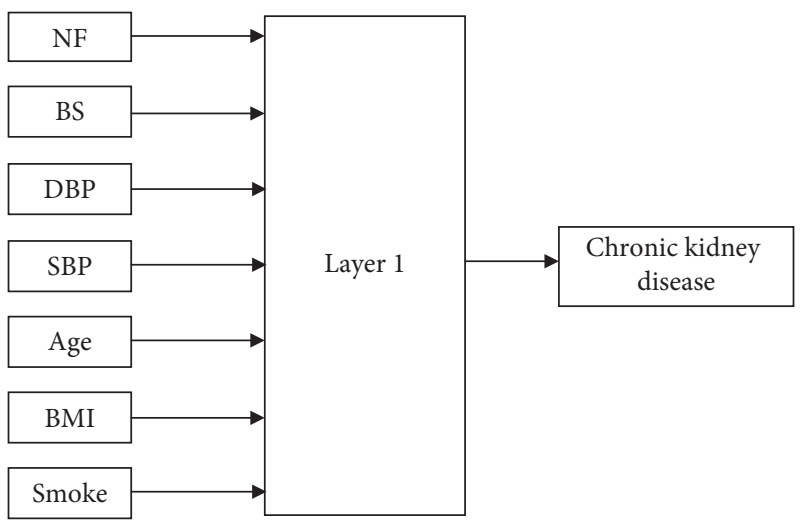

Figure 2: Proposed fuzzy expert system.

fuzzy sets. If the level of blood sugar is below 104, then it is safe, whereas it is at borderline if it is between 104 to 150 and if the level is greater than 140 then the existence level of glucose in blood is high.

4.1.3. Diastolic Blood Pressure. The diastolic blood pressure (DBP) is the function of congesting the heart with blood in between the muscle convulsion. It consists of 4 fuzzy sets. If the range is less than 89 , then the diastolic blood pressure is low. If the range is between 87 to 110 , then it is medium and if the range is between 106 to 121, then it is high. Nevertheless, if the range is above 118, then the diastolic blood pressure is extremely high.

4.1.4. Systolic Blood Pressure. When the heart contracts, the systolic blood pressure (SBP) is stimulated. It is peculiarly the utmost arterial pressure during shrinkage of heart's left ventricle. The time at which ventricular contraction occurs is called systole. If the range of systolic blood pressure is below 134 , then it is low; if the value is between 127 to 153 , then it is medium; if the range is between 142 to 172 , then it is high; and if the range is more than 162, then it is extremely high.
4.1.5. Age. Patient's kidneys are devilishly affected by age. It includes three variables. If the age is under 36 , then the patient is young; if age is within 33 to 66 , the patient is considered as the mid-age person; and if the age is greater than 52, the patient is classified as old.

4.1.6. Body Mass Index (BMI). BMI is a simple and clinically useful data for CKD diagnosis. It is calculated by dividing the person's weight with square of height. It has four variables. A person is underweight if $\mathrm{BMI}$ is less than 19 , normal weight if it is between 18.5 to 24.9 , overweight if the value is 24.6 to 30 , and obese if it is 29.5 or more.

4.1.7. Smoke. Smoking plays a major role in disturbing the kidney. It has three fuzzy sets. It is low if the range is less than 2.64, medium if the range lies between 1.8 to 9.5 , and high if the range is greater than 8.5 .

The output variable evaluates the condition of patient's kidney according to the behaviour of the input variables. The range of the output is from 0 to 10 . The value 0 means that there is no hiccup in the patient's kidney and 10 means the patient is abundantly sick. This variable consists of six fuzzy sets.

(i) Healthy: The patient is healthy if the value of the output variable is less than 1.7.

(ii) Concerning: The condition of the patient's kidney is concerning if the value of the output variable is within 1.5 to 3.26 .

(iii) Very concerning: If the range of output variable falls between 2.84 to 4.5 , then the condition of kidney is considered as "very concerning".

(iv) Sick: Sick class is assigned to the patients who get output value between 4 to 6 .

(v) Very sick: The patient is considered as very sick if the range of output variable is within 5.5 to 7.98.

(vi) Extremely Sick: The condition of the patient's kidney is extremely sick if the output value is above 7.5 .

4.2. Architecture for Input and Output Parameters. There are two ways to develop the architecture of input and output parameters:

(1) The first way is to determine the ranges of variables such as nephron functionality, blood sugar, and BMI used in fuzzy set using the confidence intervals (CI) [46].

(2) The second way is to get them from the experts or Clinical Practice Guidelines (CPG).

4.2.1. Development of the Parameters Using CIs. CIs are used on the sample of individuals from the target population because it is not possible to perform a study on entire population [39]. Sample data of 102 patients are taken, and to further obtain some important information from the 
given sample, it is crucial to apply tests. Figures 3-5 depict the normality tests applied on the dataset with Minitab 18 software. The normality tests used for this analysis are Anderson Darling and Ryan-Joiner normality test. To accept the null hypothesis, the $P$-value must be greater than 0.05 , whereas value of correlation coefficient must be near to 1 .

After the normality test, the next step is to apply confidence intervals to these three variables, i.e., Nephron Functionality, Blood sugar, and BMI. For example, in Table 1 the case of nephron functionality is mentioned, and the various parameters like mean are described as per interest.

4.2.2. Ranges of Input and Output Parameters. An efficient analysis of each variable range determines the parameters corresponding to each variable. Figure 6 shows the membership function of the nephron functionality. Figure 7 shows the membership function of blood sugar by CIs and Figure 8 shows the membership function for BMI by CIs. With the information of the nephron functionality variable, the mathematical representation of the interval used in this model can be described as follows:

$$
\begin{aligned}
\mu_{\text {very-risky }}(x) & = \begin{cases}1, & x<0.28, \\
\frac{0.35-x}{0.07}, & 0.28<x \leq 0.35,\end{cases} \\
\mu_{\text {moderately-risky }}(x) & = \begin{cases}0, & x<0.3, \\
\frac{x-0.3}{0.07}, & 0.3 \leq x \leq 0.37, \\
1, & 0.37 \leq x \leq 0.42, \\
\frac{x-0.42}{0.08}, & 0.42 \leq x \leq 0.5, \\
0, & x>0.5,\end{cases} \\
\mu_{\text {Safe-zone }}(x) & = \begin{cases}\frac{x-0.47}{0.07}, & 0.47 \leq x \leq 0.54, \\
1, & x>0.54 .\end{cases}
\end{aligned}
$$

4.3. Knowledge Base (IF-THEN Rules Formulation). In this phase, a group of rules is related to the fuzzy variables with the output classification. A knowledge base contains these fuzzy rules. The precedent-consequential way is followed for these rules. In this model, the Mamdani fuzzy rule-based model is applied, and these rules are stated as follows:

IF......Clinical variable 1 AND Clinical variable 2.... THEN. .....Output.

Example 1, inference rule no. 1.

If Nephron Functionality is moderately risky,

And Blood sugar is high,

And DBP is high,

And SBP is high,

And Age is mid age,

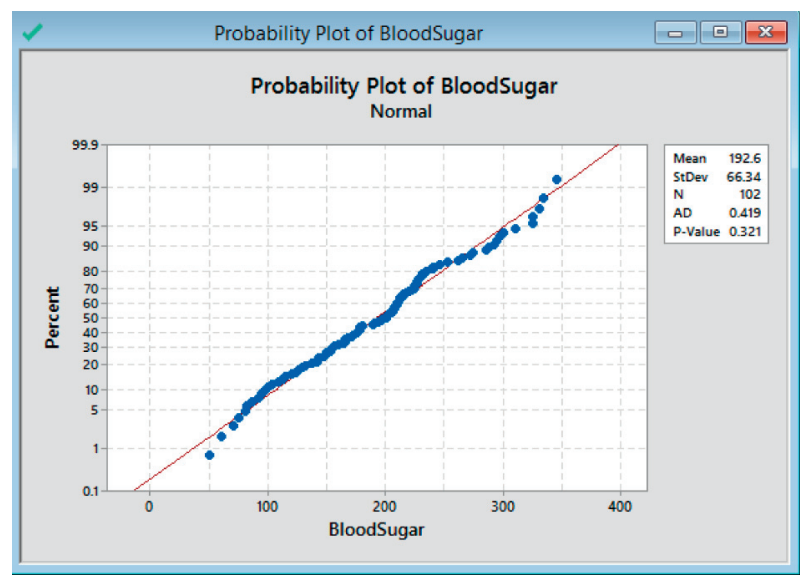

FIGURE 3: Normality test for input variable Blood Sugar (mmol/L).

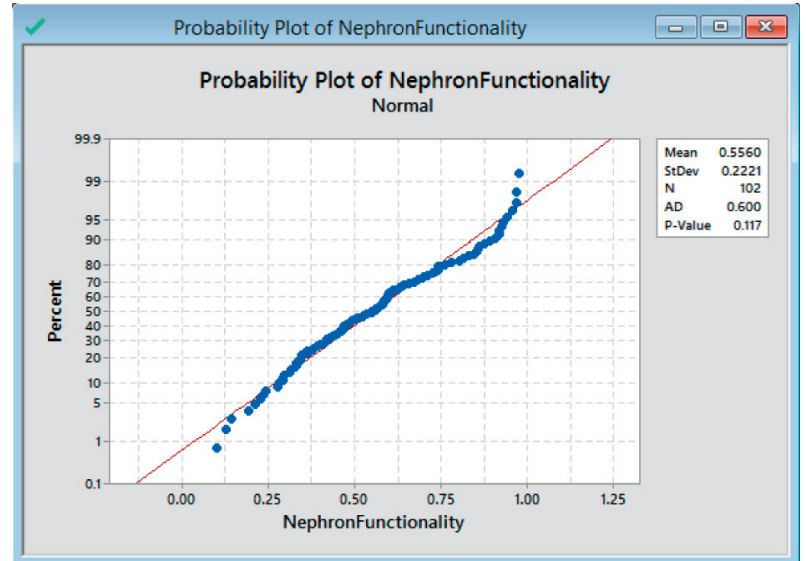

Figure 4: Normality test for input variable "Nephron Functionality" ( $\mathrm{ml} / \mathrm{min})$.

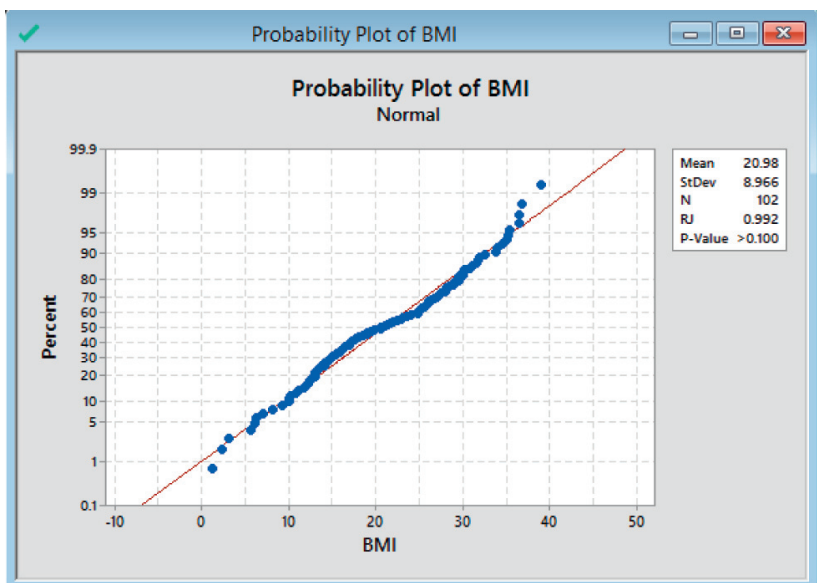

Figure 5: Normality test for input variable "BMI” $\left(\mathrm{kg} / \mathrm{m}^{2}\right)$.

And BMI is underweight,

And Smoke is Medium,

THEN the patient's kidney condition is very concerning.

Example 2, inference rule no. 81. 
TABLE 1: Parameters of nephron functionality.

\begin{tabular}{|c|c|c|c|c|c|c|c|c|c|c|}
\hline Linguistic labels & Sample size & Mean & $\sigma$ & $\alpha$ & $\mathrm{t} \alpha, n-1$ & $\mathrm{Z} \alpha=$ & Lower limit & Unilateral lower limit & Unilateral upper limit & Upper limit \\
\hline Safe-zone & 36 & 0.71 & 0.14 & 0.1 & & 1.65 & 0.478 & 0.538 & 0.548 & 1.00 \\
\hline Moderately risky & 30 & 0.40 & 0.05 & 0.1 & 2.045 & & 0.3 & 0.37 & 0.42 & 0.5 \\
\hline Very-risky & 14 & 0.23 & 0.06 & 0.1 & 2.160 & & -1 & 0.1 & 0.28 & 0.35 \\
\hline
\end{tabular}

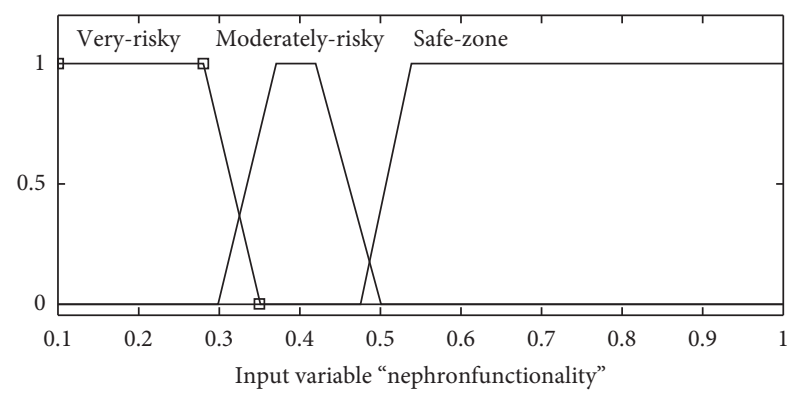

FIGURE 6: Membership function for nephron functionality by CIs.

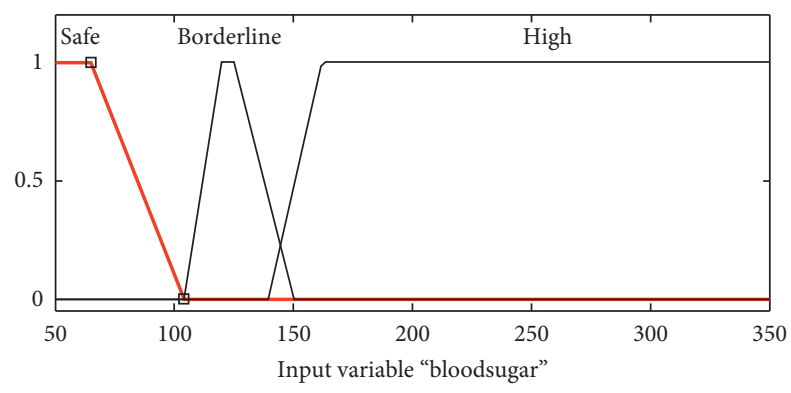

FIGURE 7: Membership function for blood sugar by CIs.

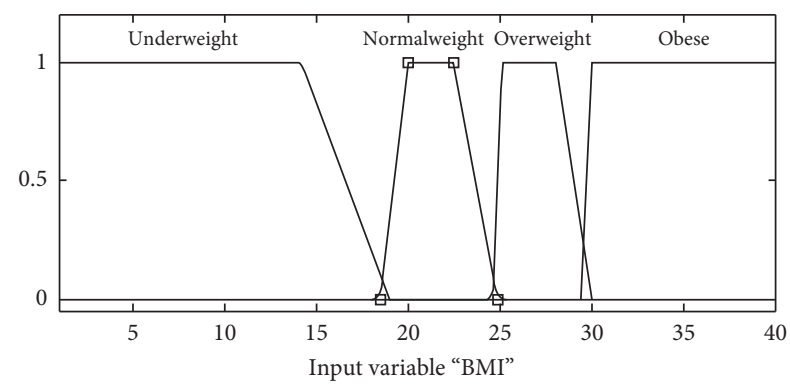

Figure 8: Membership function for BMI by CIs.

IF Nephron functionality is Very-Risky,

And Blood sugar is High,

And DBP is Extremely High,

And SBP is Extremely High

And age is Old,

And BMI is obese,

And smoke is high,

THEN patient's kidney is extremely sick.

Table 2 shows the variables in FES, and the abovementioned rules are interpreted into fuzzy rules as presented in Table 3.

In the fuzzy inference system, the input-output rules play an important role. The efficiency of a proposed system is directly proportional to the $\mathrm{I} / \mathrm{O}$ rules generated and stored in the knowledge base of expert system. Figure 9 presents the framework of knowledge base in which the rules are stored.

In the proposed model, there are seven variables; hence, it gives 5184 rules, but it is difficult to handle so many rules. Therefore, in this work, 83 rules are used. These rules have no omission because each of them has probability of its occurrence. The variables are chosen in such a way that the redundancy in rules can be avoided and impeded the dependency of one variable on other so that it will not affect the inference mechanism. The rules are also revised to find out the inconsistent rules, and the result of analysis showed positive outcomes. The abovementioned seven input variables are mixed and according to the reference rules the output is determined.

$$
\begin{aligned}
\text { IRT }= & \text { nephron functionality }(3) \times \text { blood sugar }(3) \\
& \times \text { diastolic blood pressure }(4) \\
& \times \text { systolic blood pressure }(4) \times \text { age }(3) \\
& \times \operatorname{BMI}(4) \times \operatorname{smoke}(3)=5,184 \text { inference rules. }
\end{aligned}
$$

where IRT stands for Inference rules total.

The defuzzification method computes the centre of gravity of images that is driven at the breakdown time of every input linguistic parameter. It implies that this method inspects the relationship between the degrees of membership of two joint sets and enumerates the outside of each subsequent image. The classic structure is utilized in the centroid method corresponding to the abscissa axis. The centre of gravity is in the middle of its base, in rectangles. Further, for triangles, it is set at the third part of its base as indicated by inverse side of the point inducted by hypotenuse and base. Finally, to obtain the total centroid, the summation of surface product of every figure is divided by its centroid into the total surface. [46].

4.4. Tests. Here, the need was to interview the patients suffering from CKD. The proposed fuzzy expert system is validated. This validation is done by various specialist doctors and experts from the nephrology and urology department. To evaluate confidence of the system, eighty tests are performed. Results are of a high certainty degree.

The main aim of the evaluation of system is to identify the error percentage and imperfections which could be possibly found in the fuzzy expert system. It also helped to demonstrate the expectations that will be fulfilled by system and the possible error which further helped to calculate 
TABLE 2: Variables in FES.

\begin{tabular}{|c|c|c|c|}
\hline Input variables & Linguistic label & Membership function & Interval \\
\hline \multirow{3}{*}{ Nephron functionality } & Very-risky & Trapezoidal & $(-1,0.1,0.28,0.35)$ \\
\hline & Moderately risky & Trapezoidal & $(0.3,0.37,0.42,0.5)$ \\
\hline & Safe-zone & Trapezoidal & $(0.47,0.53,0.54,1)$ \\
\hline \multirow{3}{*}{ Blood sugar } & Safe & Trapezoidal & $(-58,4,65,104)$ \\
\hline & Borderline & Trapezoidal & $(104,120,125,150)$ \\
\hline & High & Trapezoidal & $(140,162,362,458)$ \\
\hline \multirow{4}{*}{ Diastolic blood pressure } & Low & Trapezoidal & $(65,78.33,82,89)$ \\
\hline & Medium & Trapezoidal & $(87,95,100,110)$ \\
\hline & High & Trapezoidal & $(106,110,115,121)$ \\
\hline & Extremely high & Trapezoidal & $(118,123,131.7,144.9)$ \\
\hline \multirow{4}{*}{ Systolic blood pressure } & Low & Trapezoidal & $(29.6,74.4,118,134)$ \\
\hline & Medium & Trapezoidal & $(127,137,143,153)$ \\
\hline & High & Trapezoidal & $(142,157,162,172)$ \\
\hline & Extremely high & Trapezoidal & $(162,180,240,250)$ \\
\hline \multirow{3}{*}{ Age } & Young & Trapezoidal & $(-18.8,6.8,28,36)$ \\
\hline & Mid-age & Trapezoidal & $(33,44,55,66)$ \\
\hline & Old & Trapezoidal & $(52,61,93.2,118.8)$ \\
\hline \multirow{4}{*}{ BMI } & Underweight & Trapezoidal & $(-46.66,1,14.18,19)$ \\
\hline & Normal weight & Trapezoidal & $(18.5,20,22.5,24.9)$ \\
\hline & Overweight & Trapezoidal & $(24.6,25.1,28.1,30)$ \\
\hline & Obese & Trapezoidal & $(29.5,30,41,55)$ \\
\hline \multirow{3}{*}{ Smoke } & Low & Trapezoidal & $(-7.25,-0.85,1.5,2.64)$ \\
\hline & Medium & Trapezoidal & $(1.8,5.2,5.8,9.5)$ \\
\hline & High & Trapezoidal & $(8.5,11,20.8,27.2)$ \\
\hline \multicolumn{4}{|l|}{ Output variable } \\
\hline \multirow{6}{*}{ Result } & Healthy & Trapezoidal & $(-3.57,-0.34,1.1,1.7)$ \\
\hline & Concerning & Triangular & $(1.5,2.4,3.26)$ \\
\hline & Very-concerning & Triangular & $(2.84,3.67,4.5)$ \\
\hline & Sick & Triangular & $(4,5,6)$ \\
\hline & Very sick & Triangular & $(5.5,6.74,7.98)$ \\
\hline & Extremely sick & Trapezoidal & $(7.5,8.4,300)$ \\
\hline
\end{tabular}

TABLE 3: The system knowledge base.

\begin{tabular}{lcccccccc}
\hline Rule no. & Nephron functionality & Blood sugar & DBP & SBP & Age & BMI & Smoke & Conclusion \\
\hline 2 & Extremely risky & High & High & Extremely high & Mid age & Obese & Low & Very concerning \\
11 & Safe-zone & Borderline & Medium & Medium & Young & Overweight & High & Healthy \\
18 & Moderately risky & Borderline & High & High & Young & Underweight & Low & Healthy \\
29 & Very-risky & Safe & Medium & Medium & Young & Obese & High & Concerning \\
36 & Moderately risky & Borderline & Extremely high & Extremely high & Old & Underweight & Low & Very concerning \\
48 & Safe-zone & High & Extremely high & Extremely high & Mid-age & Under-weight & Medium Very concerning \\
54 & Very-risky & Safe & Extremely high & Extremely high & Young & Obese & Low & Very sick \\
64 & Very-risky & Borderline & High & High & Mid-age & Overweight & High & Very-sick \\
73 & Moderately risky & High & Extremely high & Extremely high & Young & Overweight & High & Extremely sick \\
80 & Moderately risky & High & Extremely high & Extremely high & Old & Obese & High & Extremely sick \\
\hline
\end{tabular}

performance of system and where the performance measure was lacking behind, it helped to improve that problem.

The various tests that were done with this fuzzy expert system are given in Table 4. In these tests, doctors evaluated suffering patients by this fuzzy expert system with various input variables that are used in the system, and then the obtained results were compared with the actual results. In Test 1 , Nephron functionality is equal to 0.458 , blood sugar is 132.2, Diastolic Blood Pressure is equal to 105.5, systolic Blood Pressure equal to 128.9 , age is 38 , BMI have the value 29.55 , and smoke value is 2.037 , and then control assessment by expert system for that particular patient's kidney condition is concerning. The result as per doctor is also that the kidney of the patient is in concerning phase.

4.5. Response Surface Analysis. The system also gives the three-dimensional plot which describes the influence of input variables on output and output can also be calculated at particular inputs from this three-dimensional plot. These surfaces are helping to depict the level of clinical variables that influence results. It can be seen as if the 


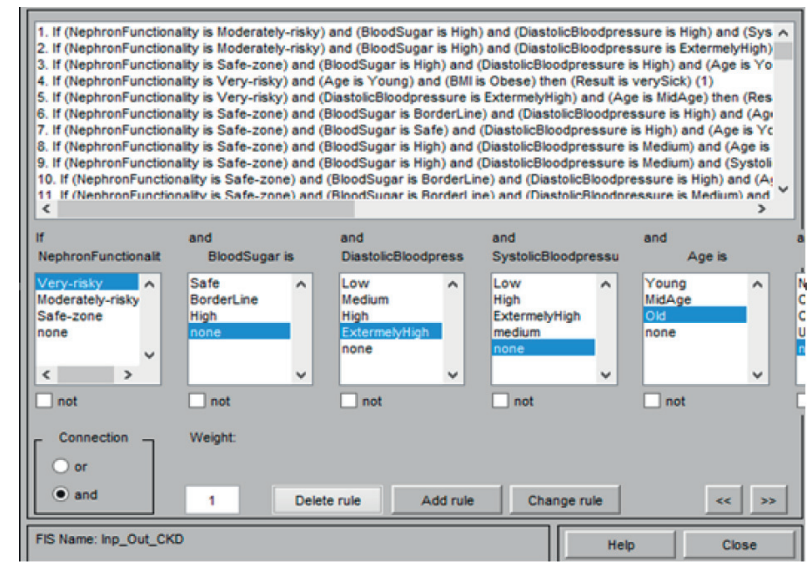

FIgURE 9: Input-output rules for the proposed expert system.

combinations of variables are in light colour, then it means the optimal zone, whereas if the colour of combination is dark, then the variable levels activate a nonoptimal zone, which indicates a possibility of having CKD to a particular patient.

\section{Experimental Results and Analysis}

Eighty tests were carried out to analyse CKD using developed fuzzy expert system. The team of doctors also diagnosed each patient and gave result for same. After that, the results given by experts are compared with obtained results from the fuzzy expert system. By correlating the two results, 75 out of 80 tests were correctly classified, and hence the system showed 5 errors. A sample of tests is shown in Table 4.

In Table 5, the confusion matrix is given. 80 cases of different patients are carried out. In the first column, 15 cases of healthy patients are considered and all of them are classified into correct class, i.e., healthy class. In the second column, the data of 10 patients at the concerning level is considered, but as a result out of 10 cases, 8 cases are exactly classified into concerning class and rest of 2 are classified into sick class. Similarly, in the third column, 16 cases very concerning are carried out, and from those 16 cases 15 are classified correctly and one is classified into class sick. In the fourth column, out of 11 diagnosed patients, 1 is classified incorrectly and 10 are in exact class. Likewise, fifth column illustrates that 14 diagnosed patients of class very sick are classified in correct class. At last, the sixth column shows that there are 14 cases of extremely sick patient, but out of 14,1 is misclassified.

$$
\text { confidence indicator }=\left(\frac{\text { success number }}{\text { total number of tests }} * 100\right) \text {, }
$$

where number of successes $=75$ and total number of tests $=80$.

It was examined that $93.75 \%$ of the results given by fuzzy expert system are classified correctly by calculating the confidence indicator using above formula. Therefore, by observing the confidence indicator, it can be said that the developed fuzzy expert system can work as the supporting tool for doctors in diagnosis of CKD.

The results show that the categorization by fuzzy inference system for CKD is $06.25 \%$ wrong. Now, the first three classes that is healthy, concerning, and very concerning are taken as "no". Similarly, the rest of classes sick, very sick, and extremely sick are taken as "yes". Hence, the confusion matrix shown in Table 4 is reduced to $2 * 2$ matrix and shown in Table 6.

The performance of developed medical expert system is evaluated by considering various parameters like accuracy, precision, specificity, and sensitivity.

In case of CKD, from Table 5,

(i) TP: true positive is 37

(ii) FN: false negative is 02

(iii) FP: false positive is 03

(iv) TN: true negative is 38

$$
\begin{aligned}
\text { ensitivity } & =\frac{(\mathrm{TP})}{(\mathrm{TP}+\mathrm{FN})}=\frac{37}{37+02}=94.87 \%, \\
\text { specificity } & =\frac{(\mathrm{TN})}{(\mathrm{TN}+\mathrm{FP})}=\frac{38}{38+03}=92.68 \%, \\
\text { precision } & =\frac{(\mathrm{TP})}{(\mathrm{TP}+\mathrm{FP})}=\frac{37}{37+03}=92.5 \%, \\
\text { classification accuracy } & =\frac{(\mathrm{TP}+\mathrm{TN})}{(\mathrm{TP}+\mathrm{FP}+\mathrm{TN}+\mathrm{FN})} \\
& =\frac{37+38}{37+03+38+02}=93.75 \% .
\end{aligned}
$$

These calculated parameters are shown in the form of graph in Figure 10.

The main aim to develop this expert system is that it will help doctors to identify the CKD in patients. This medical 
TABLE 4: A sample of tests performed with developed fuzzy expert system.

\begin{tabular}{|c|c|c|c|c|c|c|c|c|c|c|}
\hline Test & Nephron functionality & Blood sugar & $\mathrm{DBP}$ & SBP & Age & BMI & Smoke & CADT & CAFES & Success \\
\hline 1 & 0.458 & 132.1 & 105.5 & 128.9 & 38 & 29.55 & 2.037 & Concerning & Concerning & Yes \\
\hline 2 & 0.226 & 165 & 113 & 155 & 64 & 3 & 10 & Extremely sick & Extremely sick & Yes \\
\hline 3 & 0.1 & 300 & 120 & 212 & 26 & 35 & 20 & Extremely sick & Very sick & No \\
\hline 4 & 0.4083 & 75.47 & 89.72 & 134.2 & 20 & 25.92 & 8.333 & Healthy & Healthy & Yes \\
\hline 5 & 0.875 & 177.4 & 107.3 & 152.6 & 59 & 17.25 & 16.48 & Concerning & Concerning & Yes \\
\hline 6 & 0.3292 & 81.13 & 121 & 180.7 & 24 & 30.07 & 2.075 & Very sick & Very sick & Yes \\
\hline 7 & 0.5917 & 137.7 & 115.6 & 144.7 & 28 & 26.24 & 18.33 & Healthy & Healthy & Yes \\
\hline 8 & 0.6417 & 205.7 & 120.3 & 176.4 & 47 & 13.64 & 6.481 & Very sick & Very concerning & No \\
\hline 9 & 0.9417 & 86.79 & 93.43 & 139.4 & 58 & 10.03 & 8.333 & Healthy & Healthy & Yes \\
\hline 10 & 0.3583 & 120.8 & 124 & 192.3 & 27 & 27.36 & 12.41 & Concerning & Concerning & Yes \\
\hline 11 & 0.4583 & 86.79 & 104.5 & 139.4 & 64 & 25.92 & 9.444 & Concerning & Concerning & Yes \\
\hline 12 & 0.2083 & 109.4 & 115.6 & 160.6 & 56 & 35.31 & 11.67 & Extremely sick & Extremely sick & Yes \\
\hline 13 & 0.975 & 324.5 & 111 & 184.3 & 28 & 27.36 & 9.381 & Healthy & Healthy & Yes \\
\hline 14 & 0.425 & 177.4 & 109.2 & 155.3 & 68 & 12.19 & 6.481 & Very concerning & Very concerning & Yes \\
\hline 15 & 0.5917 & 137.7 & 115.6 & 144.7 & 28 & 26.64 & 18.33 & Healthy & Healthy & Yes \\
\hline 16 & 0.4622 & 178 & 122.1 & 180.7 & 61 & 29 & 17.32 & Extremely sick & Extremely sick & Yes \\
\hline 17 & 0.3292 & 81.13 & 121 & 180.7 & 24 & 30.07 & 2.075 & Very sick & Very sick & Yes \\
\hline 18 & 0.3462 & 154.7 & 108.6 & 150 & 56 & 15.35 & 3.208 & Sick & Sick & Yes \\
\hline 19 & 0.4991 & 284.9 & 120.1 & 176.4 & 40 & 30.07 & 1.698 & Sick & Very concerning & No \\
\hline 20 & 0.4841 & 148.8 & 87.93 & 136.3 & 30 & 17.65 & 3.659 & Concerning & Concerning & Yes \\
\hline 21 & 0.55 & 86.79 & 91.79 & 134.2 & 62 & 16.08 & 5.849 & Healthy & Healthy & Yes \\
\hline 22 & 0.4311 & 160.4 & 85.19 & 128.9 & 90 & 3.22 & 19.06 & Sick & Sick & Yes \\
\hline 23 & 0.3972 & 132.1 & 88.02 & 134.2 & 36 & 25.65 & 1.321 & Healthy & Healthy & Yes \\
\hline 24 & 0.55 & 149.1 & 108.8 & 147.4 & 54 & 17.56 & 10.38 & Concerning & Concerning & Yes \\
\hline 25 & 0.3462 & 81.13 & 85.19 & 126.2 & 42 & 30.8 & 10 & Healthy & Very concerning & No \\
\hline 26 & 0.55 & 171.7 & 97.45 & 102.5 & 31 & 27 & 14.91 & Healthy & Healthy & Yes \\
\hline 27 & 0.4651 & 154.7 & 105.9 & 102.5 & 28 & 10.02 & 8.491 & Healthy & Healthy & Yes \\
\hline 28 & 0.3292 & 149.1 & 123.9 & 181.7 & 24 & 31.54 & 10 & Extremely sick & Extremely sick & Yes \\
\hline 29 & 0.343 & 147.1 & 120.7 & 172 & 60 & 31.03 & 10.6 & Extremely sick & Extremely sick & Yes \\
\hline 30 & 0.361 & 111.8 & 107.9 & 163.7 & 39 & 24.79 & 2.2 & Healthy & Healthy & Yes \\
\hline 31 & 0.487 & 111.8 & 107.9 & 155.5 & 34 & 13.09 & 2.2 & Concerning & Healthy & No \\
\hline 32 & 0.343 & 141.2 & 117.7 & 155.5 & 31 & 17.77 & 1 & Healthy & Healthy & Yes \\
\hline
\end{tabular}

DBP: diastolic blood pressure, SBP: systolic blood pressure, CADT: control assessment by doctors' team, and CAFES: control assessment by fuzzy expert system.

TABLE 5: Confusion matrix for CKD.

\begin{tabular}{lcccccc}
\hline Healthy & Concerning & Very concerning & Sick & Very sick & Extremely sick & Class names \\
\hline 15 & 00 & 00 & 00 & 00 & 00 & 00 \\
00 & 08 & 00 & 00 & 02 & 00 & Healthy \\
00 & 00 & 15 & 01 & 00 & 00 & Very concerning \\
01 & 00 & 00 & 10 & 00 & 00 & Sick \\
00 & 00 & 00 & 00 & 14 & 13 & Very sick \\
00 & 00 & 01 & 00 & 00 & & Extremely sick \\
\hline
\end{tabular}

expert system is able to diagnose the disease and can help specialist to provide an appropriate and suitable treatment for disease.

To portray the behaviour of two given variables in 3dimensional surface, the response surface is used. It explains the probability of risk with which a person suffers on two considered variables. Three plots are considered in this work. First in Figure 11, it is the plot between BMI and nephron functionality. The response surface plot of variable BMI and nephron functionality is analysed by test 2 in which the value of BMI is 35 and similarly value of nephron functionality is 0.1 . From plot, it can be estimated easily that at these values, the result is high i.e. extremely sick.
TABLE 6: Matrix with reduced dimensionality.

\begin{tabular}{lcc}
\hline Yes & No & Class name \\
\hline 37 & 02 & Yes \\
03 & 38 & No \\
\hline
\end{tabular}

Furthermore, by Figures 12 and 13, the output can be estimated by considering blood sugar with nephron functionality and BMI with blood sugar, respectively. With a complete analysis of all the potential instances of test two response surface plots, and with grouping of seven parameters, it is found that the parameters with most extreme impact in diagnosis of CKD are nephron functionality, blood sugar, and BMI. These three variables have the highest effect 


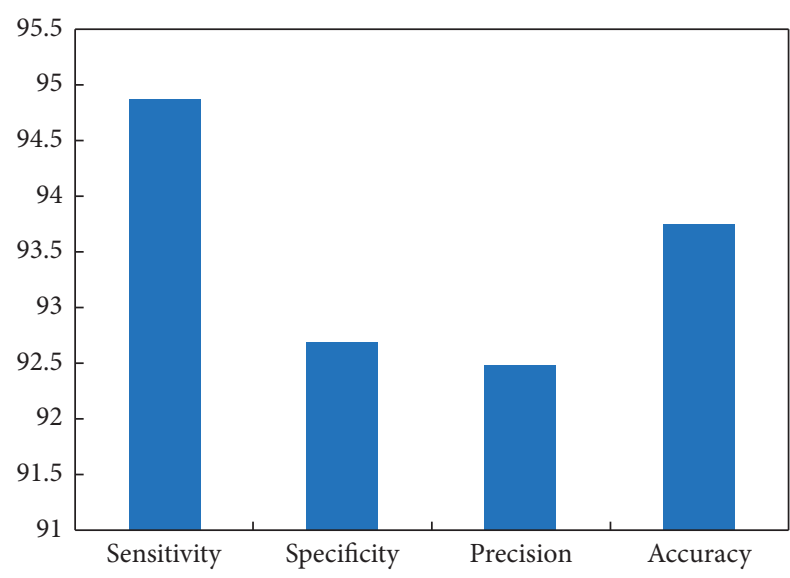

Figure 10: Performance of proposed system.

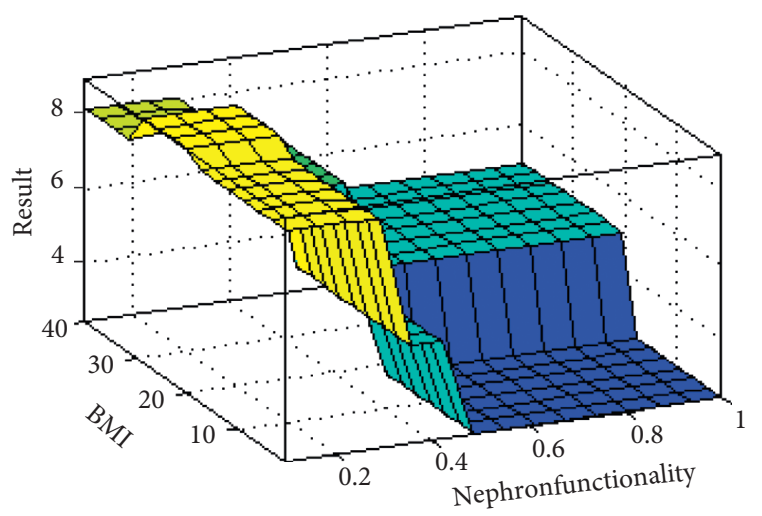

FIGURE 11: Response surface plot on test 2 with BMI and nephron functionality.

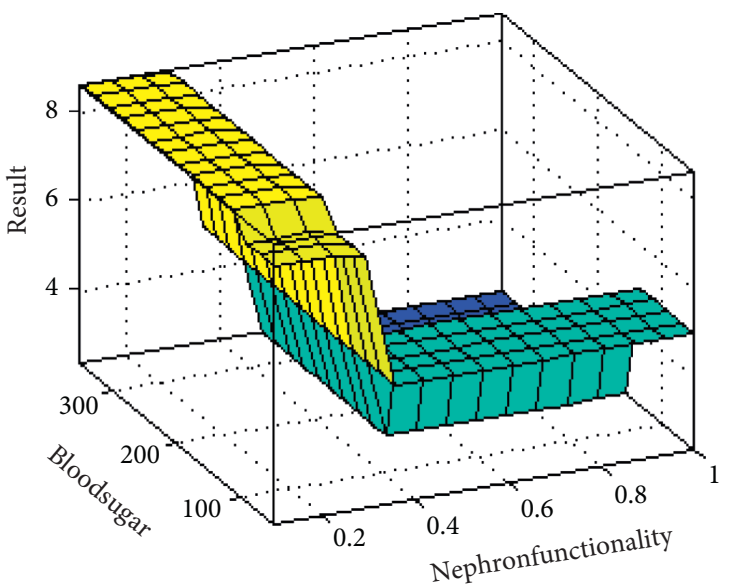

Figure 12: Response surface plot on test 2 with blood sugar and nephron functionality.

on patient's health in case of CKD. Therefore, doctors must give appropriate recommendation to patients to make their health stable and be cured in time from this CKD.

Figure 14 shows the input values of various input variables used in medical expert system for diagnosis of CKD and output corresponding to given inputs. Image-processing

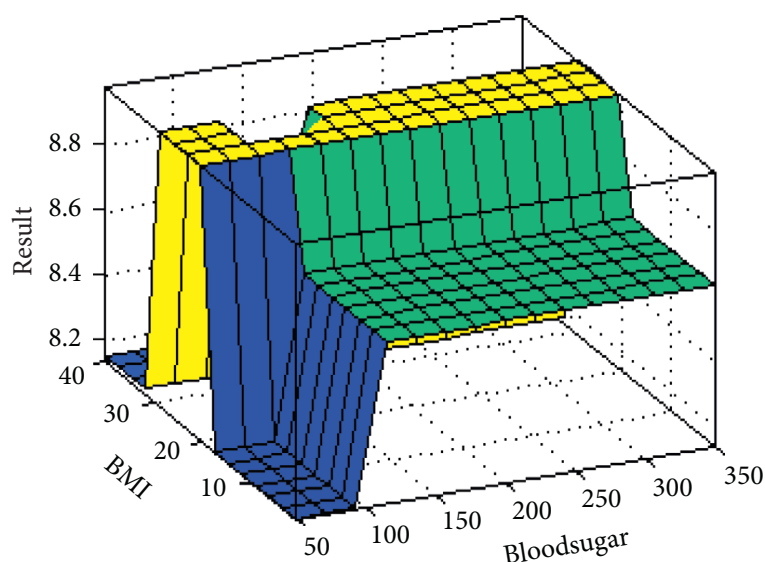

FIgURE 13: Response surface plot on test 2 with BMI and blood sugar.

techniques such as registration of an image, segmentation of an image, and extraction of features have also been applied on acquired magnetic resonance images and computed tomography images.

Additionally, the artificial neural network techniques such as backpropagation neural network and modular neural network are also used for the detection of CKD. Moreover, many systems have been proposed by using machine learning algorithms for the diagnosis of CKD such as $k$ star and $k$ nearest neighbor classifier to classify the stage of the disease into different classes according to the symptoms and risk factors.

All these abovementioned techniques deal with the crisp numbers. The developed system in this work deals with the fuzzy values, and it has also given more accuracy than these techniques.

\section{Discussion}

The developed fuzzy logic-based medical expert system is a supporting tool in decision-making about diagnosis of CKD. Although, specialist doctor's decision will be considered as final, the developed medical expert system can also make decision as a specialist doctor. Hence, it could be a very useful tool for assisting medical doctors for the robust decision regarding the $\mathrm{CKD}$.

The fuzzy expert system can also be suitable in remote areas, such as villages, and small towns, as there is always scarcity of specialists in such places. This expert system can also help to train the new doctors who do not have enough experience to diagnose the CKD, because this fuzzy expert system has knowledge and information stored in knowledge base that was acquired from specialists of the disease. Hence, it can be used as a training tool as well.

The accuracy obtained from the developed system is $93.75 \%$. It means the system is successful in identification of $93.75 \%$ of the total cases considered in this research work. Another performance parameter considered in this work is precision. The precision obtained from this developed system is $92.5 \%$. It means the developed system is having high quality of giving exact and clear results. Similarly, the 


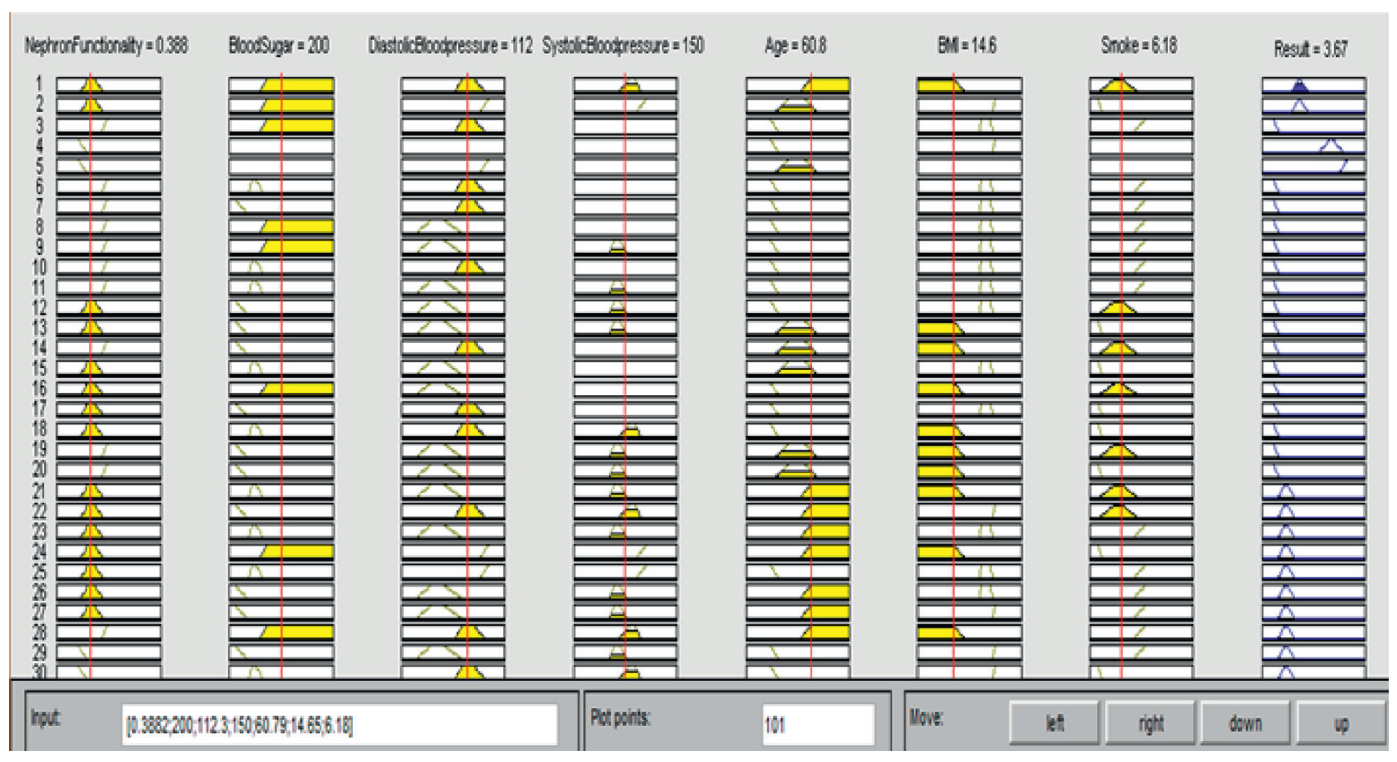

FigURE 14: Mapping of rules for output.

sensitivity and specificity of developed system are $94.87 \%$ and $92.68 \%$. It means developed system is capable of measuring the proportion of actual positives and negatives which are correctly determined as such.

The whole work done in this study is new and original as the clinical tests, and risk factors considered in this study have not been incorporated in the systems similar to it. The various research works are discussed in Introduction to understand more about risk factors, prevention, and treatment of CKD. Competitive advantage is that the proposed expert system is able to diagnose the disease with various variations in input variables. Variation states considered in this work are healthy, concerning, very concerning, sick, very sick and extremely sick.

The main contribution of this work is that it is a supporting tool which is capable to make decisions, or it supports the decision-making process according to the given inputs to system by the user to identify whether the patient is suffering from CKD or not. To improve the success rate of fuzzy expert system, the recommendation is to gather more knowledge about the disease from specialist doctor and make rules as enough as possible. One can also increase the number of variables used as input to recognize the CKD and make their range accordingly so that the result can be calculated more accurately.

\section{Conclusion}

The diagnosis of chronic kidney disease is a difficult task. There is always some possibility of misdiagnosis that leads to wrong treatment. This work proposes a fuzzy expert system that can help a doctor and nonspecialist to examine the chronic kidney patient.

The fuzzy expert system is based on clinical test ranges. It works on two types of information, that is, the knowledge acquired from specialist doctors and experts from the nephrology and urology departments. This study also gives response surface plot of various input variables used to recognize the disease. This system can give correct results about the health status of a patient.

In the current state, it would be inappropriate to say that the developed fuzzy expert system will replace the specialist doctors or knowledge of the team of doctors. However, this could be a supporting tool for doctors that could assist in decision-making and does not replace their admirable work. In addition, only computer and software are required to implement this medical expert system. Therefore, this system can be used in hospitals, where there is lack of resources and can be used in those geographic areas where hospitals are not available.

\section{Data Availability}

The data are available on request on demand through the first author jimmy.21733@lpu.co.in. or via phone +9779288330.

\section{Conflicts of Interest}

The authors declare that there are no conflicts of interest regarding the publication of this paper.

\section{Acknowledgments}

This work was supported by Institute of Information and Communications Technology Planning and Evaluation (IITP) grant funded by the Korea Government (MSIT) (no.2018-0-01369).

\section{References}

[1] S. Varughese and G. Abraham, "Chronic kidney disease in India: a clarion call for change," Clinical Journal of the American Society of Nephrology, vol. 13, no. 5, pp. 802-804, 2018. 
[2] S. Kutia, S. H. Chauhdary, C. Iwendi, L. Liu, W. Yong, and A. K. Bashir, "Socio-technological factors affecting user's adoption of eHealth functionalities: a case study of China and Ukraine eHealth systems," IEEE Access, vol. 7, pp. 9077790788, 2019.

[3] G. P. Joshi, S. Acharya, C.-S. Kim, B.-S. Kim, and S. W. Kim, "Smart solutions in elderly care facilities with RFID system and its integration with wireless sensor networks," International Journal of Distributed Sensor Networks, vol. 10, no. 8, Article ID 713946, 2014.

[4] S. Varughese, "Chronic kidney disease in India a clarion call for change," Clinical Journal of the American Society of $\mathrm{Ne}$ phrology, vol. 13, no. 5, pp. 802-804, 2018.

[5] Y. Kubota, Y. Mitsukura, M. Fukumi, N. Akarnatsu, and M. Yasutomo, "Automatic extraction of a kidney region by using the q-1eaning," in Proceedings of 2004 International Symposium on Intelligent Signal Processing and Communication Systems, 2004. ISPACS 2004, pp. 536-540, IEEE, Seoul, South Korea, November 2004.

[6] D. Turco, C. Corsi, S. Severi, and R. Mignani, "Assessment of kidney volumes in Polycystic Kidney Disease from Coronal and Axial MR Images," in Proceedings of the 2013 8th International Symposium On Image And Signal Processing And Analysis (ISPA), pp. 528-532, IEEE, Trieste, Italy, September 2013.

[7] H. Tang, J. L. Dillenseger, and L. M. Luo, "Intra subject 3D/3D kidney registration using local mutual information maximization," in Proceedings of the 2007 29th Annual International Conference of the IEEE Engineering in Medicine and Biology Society, pp. 6379-6382, IEEE, Lyon, France, August 2007.

[8] K. Bommanna Raja and M. Madheswaran, "Determination of kidney area independent unconstrained features for automated diagnosis and classification," in Proceedings of the 2007 International Conference on Intelligent and Advanced Systems, pp. 724-729, Kuala Lumpur, Malaysia, 2007.

[9] F. Iqbal, A. S. Pallewatte, and J. P. Wansapura, "Texture analysis of ultrasound images of chronic kidney disease," in Proceedings of the 17th International Conference on Advances in ICT for Emerging Regions, ICTer 2017, pp. 299-303, IEEE, Colombo, Sri Lanka, September 2017.

[10] R. M. Pujari and V. D. Hajare, "Analysis of ultrasound images for identification of chronic kidney," in Proceedings of the First International Conference on Networks and Soft Computing, pp. 380-383, IEEE, Guntur, India, August 2014.

[11] K. Bommanna Raja and M. Madheswaran, "Determination of kidney area independent unconstrained features for automated diagnosis and classification," in Proceedings of the 2007 International Conference On Intelligent And Advanced Systems, ICIAS, pp. 724-729, IEEE, Kuala Lumpur, Malaysia, November 2007.

[12] M. Ahmad, V. Tundjungsari, D. Widianti, P. Amalia, and U. A. Rachmawati, "Diagnostic decision support system of chronic kidney disease using support vector machine," in Proceedings of the 2 nd International Conference on Informatics and Computing, ICIC, IEEE, Kuala Lumpur, Malaysia, November 2017.

[13] W. H. S. D. Gunarathne, K. D. M. Perera, and K. A. D. C. P. Kahandawaarachchi, "Performance evaluation on machine learning classification techniques for disease classification and forecasting through data analytics for chronic kidney disease (CKD)," in Proceedings of the 2017 IEEE 17th International Conference on Bioinformatics and Bioengineering, BIBE, pp. 291-296, IEEE, Washington, DC, USA, October 2017.
[14] M. S. Wibawa, I. M. D. Maysanjaya, and I. M. A. W. Putra, "Boosted classifier and features selection for enhancing chronic kidney disease diagnose," in Proceedings of the 2017 5th International Conference on Cyber and IT Service Management, CITSM, IEEE, Denpasar, Indonesia, August 2017.

[15] U. N. Dulhare and M. Ayesha, "Extraction of action rules for chronic kidney disease using Naïve bayes classifier," in Proceedings of the 2016 IEEE International Conference on Computational Intelligence and Computing Research, ICCIC, IEEE, Chennai, India, December 2016.

[16] E. Avci, S. Karakus, O. Ozmen, and D. Avci, "Performance comparison of some classifiers on chronic kidney disease data," in Proceedings of the 6th International Symposium on Digital Forensic and Security, ISDFS 2018, IEEE, March 2018.

[17] R. K. Chiw, R. Y. Chen, S. Wang, and S. Jian, "Intelligent systems on the cloud for the early detection of chronic kidney disease," in Proceedings of the 2012 International Conference on Machine Learning and Cybernetics, IEEE, Xian, China, July 2012.

[18] V. Kunwar, K. Chandel, A. S. Sabitha, and A. Bansal, "Chronic kidney disease analysis using data mining classification," in Proceedings of the Cloud System And Big Data Engineering (Confluence), 2016 6th International Conference, IEEE, Noida, India, January 2016.

[19] T. Adam, U. Hashim, and U. S. Sani, "Designing an artificial neural network model for the prediction of kidney problems symptom through patient's metal behavior for pre-clinical medical diagnostic," in Proceedings of the 2012 International Conference on Biomedical Engineering, ICoBE 2012, IEEE, Penang, Malaysia, February 2012.

[20] L. Xun, N. Li, X. Wu, and T. Lou, "Application of radial basis function neural network to estimate glomerular filtration rate in Chinese patients with chronic kidney disease," in Proceedings of the 2010 International Conference on Computer Application and System Modeling (ICCASM 2010), IEEE, Taiyuan, China, October 2010.

[21] M. A. Venkatachalam, K. A. Griffin, R. Lan, H. Geng, P. Saikumar, and A. K. Bidani, "Acute kidney injury: a springboard for progression in chronic kidney disease," American Journal of Physiology-Renal Physiology, vol. 298, no. 5, pp. F1078-F1094, 2010.

[22] E. Yudaningtyas, D. H. Santjojo, W. Djuriatno, I. Siradjuddin, and M. R. Hidayatullah, "Identification of pulse frequency spectrum of chronic kidney disease patients measured at TCM points using FFT processing," in Proceedings of the 2017 15th International Conference on Quality in Research (QiR): International Symposium on Electrical and Computer Engineering, pp. 169-172, IEEE, Nusa Dua, Indonesia, July 2017.

[23] C.-J. Lin, C.-Y. Chen, P.-C. Wu et al., "Intelligent system to predict intradialytic hypotension in chronic hemodialysis," Journal of the Formosan Medical Association, vol. 117, no. 10, pp. 888-893, 2018.

[24] S. D. Arasu and R. Thirumalaiselvi, "A novel imputation method for effective prediction of coronary kidney disease," in Proceedings of the 2017 2nd International Conference on Computing and Communications Technologies, ICCCT, pp. 127-136, IEEE, Chennai, India, February 2017.

[25] S. R. Raghavan, V. Ladik, and K. B. Meyer, "Developing decision support for dialysis treatment of chronic kidney failure," IEEE Transactions on Information Technology in Biomedicine, vol. 9, no. 2, pp. 229-238, 2005.

[26] C. Paper and N. Dey, "Hybrid modified cuckoo search-neural network in chronic kidney disease classification hybrid modified cuckoo search-neural network in chronic kidney 
disease classification," in Proceedings of the 2017 14th International Conference on Engineering of Modern Electric Systems (EMES), pp. 164-167, IEEE, Oradea, Romania, June 2017.

[27] C. I. Bondor, I. M. Kacso, A. R. Lenghel, and A. Muresan, "Hierarchy of risk factors for chronic kidney disease in patients with type 2 diabetes mellitus," in 2012 IEEE 8th International Conference on Intelligent Computer Communication and Processing), pp. 103-106, IEEE, ClujNapoca, Romania, August 2012.

[28] A. Salekin and J. Stankovic, "Detection of chronic kidney disease and selecting important predictive attributes," in Proceedings of the 2016 IEEE International Conference on Healthcare Informatics, ICHI, pp. 262-270, IEEE, Chicago, IL, USA, October 2016.

[29] T. Shen, T. Fang, Y. Ou, and C. Wang, "Low-cost detection of cardiovascular disease on chronic kidney disease and dialysis patients based on hybrid heterogeneous ECG features including T-wave alternans and heart rate variability," in Proceedings of the 2010 Computing in Cardiology, IEEE, Belfast, UK, pp. 561-564, September 2010.

[30] R. B. Fricks, A. Bobbio, P. Orientale, and K. S. Trivedi, "Reliability models of chronic kidney disease," in Proceedings of the 2016 Annual Reliability and Maintainability Symposium (RAMS), January 2016.

[31] I. Council, S. Lanka, and A. Pradesh, "Prevalence of chronic kidney disease in India - where are we heading?" Indian Journal of Nephrology, vol. 25, no. 3, 2015.

[32] A. Batra, U. Batra, and V. Singh, "A review to predictive methodology to diagbose chronic kidney disease," in Proceedings of the 2016 3rd International Conference on Computing for Sustainable Global Development (INDIACom), IEEE, New Delhi, India, March 2016.

[33] A. Sobrinho, L. Dias Da Silva, M. E. Pinheiro, P. Cunha, A. Perkusich, and L. Medeiros, "Formal specification of a tool to aid the early dignosis of the chronic kidney disease," in Proceedings of the 2015 CHILEAN Conference on Electrical, Electronics Engineering, Information and Communication Technologies (CHILECON), pp. 173-178, IEEE, Santiago, Chile, October 2015.

[34] Q.-L. Zhang and D. Rothenbacher, "Prevalence of chronic kidney disease in population-based studies: systematic review," BMC Public Health, vol. 8, no. 1, 2008.

[35] H. Ahmadi, M. Gholamzadeh, L. Shahmoradi, M. Nilashi, and P. Rashvand, "Diseases diagnosis using fuzzy logic methods: a systematic and meta-analysis review," Computer Methods and Programs in Biomedicine, vol. 161, pp. 145-172, 2018.

[36] S. Sivathasan, F. Cecelja, and W. Balachandran, "ECG diagnosis using neural network and fuzzy expert system," in Proceedings of the 17th IEEE Instrumentation and Measurement Technology Conference [Cat. No. 00CH37066], pp. 988-992, IEEE, Baltimore, MD, USA, May 2000.

[37] E. Rovenţa and G. Roşu, "The diagnosis of some kidney diseases in a small prolog expert system," in Proceedings of the 2009 3rd International Workshop on Soft Computing Applications, SOFA, pp. 219-224, IEEE, Arad, Romania, August 2009.

[38] S. Das and P. K. Ghosh, "Hypertension Diagnosis: a comparative study using fuzzy expert system and neuro fuzzy system," in Proceedings of the 2013 IEEE International Conference on Fuzzy Systems (FUZZ-IEEE), July 2013.

[39] R. Meza-palacios, A. A. Aguilar-lasserre, E. L. Ureña-Bogarín, C. F. Vázquez-Rodríguez, R. Posada-Gómez, and A. Trujillomata, "Development of a fuzzy expert system for the nephropathy control assessment in patients with type 2 diabetes mellitus," Expert Systems With Applications, vol. 72, no. 1, pp. 335-343, 2017.

[40] G. Ahmad, M. A. Khan, S. Abbas, A. Athar, B. S. Khan, and M. S. Aslam, "Automated Diagnosis of Hepatitis B Using Multilayer Mamdani Fuzzy Inference System," vol. 2019, Article ID 6361318, 11 pages, 2019.

[41] A. Ali, L. Abbas, M. Shafiq et al., "Hybrid fuzzy logic scheme for efficient channel utilization in cognitive radio networks," IEEE Access, vol. 7, pp. 24463-24476, 2019.

[42] S. Ahmed, T. Kabir, N. T. Mahmood, and R. M. Rahman, "Diagnosis of Kidney Disease Using Fuzzy Expert System," in Proceedings of the 8th International Conference on Software, Knowledge, Information Management and Applications (SKIMA 2014), IEEE Dhaka, Bangladesh, December 2014.

[43] S. Tapaswini, C. Mu, D. Behera, and S. Chakraverty, "Solving imprecisely defined vibration equation of large membranes," Engineering Computations, vol. 34, no. 8, pp. 2528-2546, 2017.

[44] N. Chetty, K. S. Vaisla, and S. D. Sudarsan, "Role of attributes selection in classification of chronic kidney disease patients," in Proceedings of the 2015 International Conference on Computing, Communication and Security (ICCCS), IEEE, Pamplemousses, Mauritius, December 2015.

[45] A. K. Singh, Y. M. K. Farag, B.V. Mittal et al., "Epidemiology and risk factors of chronic kidney disease in India-results from the SEEK (screening and early evaluation of kidney disease) study," BMC Nephrology, vol. 14, no. 1, pp. 1-10, 2013.

[46] K. R. D. Hernández, A. A. A. Lasserre, R. P. Gómez, J. A. P. Guzmán, and B. E. G. Sánchez, "Development of an expert system as a diagnostic support of cervical cancer in atypical glandular cells, based on fuzzy logics and image interpretation," vol. 2013, Article ID 796387, 17 pages, 2013. 